\title{
Les confréries de dévotion et l'éducation de la foi
}

\section{Brigitte Caulier}

Volume 56, 1989

Changements culturels et éducation de la foi

Cultural Change and Education of the Faith

URI : https://id.erudit.org/iderudit/1006957ar

DOI : https://doi.org/10.7202/1006957ar

Aller au sommaire du numéro

Éditeur(s)

Les Éditions Historia Ecclesiæ Catholicæ Canadensis Inc.

ISSN

0318-6172 (imprimé)

1927-7067 (numérique)

Découvrir la revue

Citer cet article

Caulier, B. (1989). Les confréries de dévotion et l'éducation de la foi. Sessions d'étude - Société canadienne d'histoire de l'Église catholique, 56, 97-112.

https://doi.org/10.7202/1006957ar

\section{Résumé de l'article}

Les confréries de dévotion recrutant des adultes, telles que les congrégations mariales ou leurs copies, ont été des structures privilégiées pour soutenir l'éducation de la foi. Les exercices collectifs comportaient des périodes d'instruction et de prière ainsi que des séances d'apprentissage de la méditation. Le clergé qui dirigeait ces associations, recherchait une meilleure intériorisation de la foi et des principes de la religion chrétienne chez des laïcs déjà sélectionnés pour leur bonne conduite et leur piété. Les directeurs profitaient des assemblées pour rafraîchir les connaissances doctrinales des associés et surtout pour leur rappeler leurs devoirs de chrétiens. Les sujets d'instructions, les lectures et les conseils donnés aux confrères et aux consoeurs font apparaître la primauté accordée à la famille — parents comme enfants et même domestiques - dans l'épanouissement de la foi.
Tous droits réservés @ Les Éditions Historia Ecclesiæ Catholicæ Canadensis Inc., 1989
Ce document est protégé par la loi sur le droit d'auteur. L'utilisation des services d'Érudit (y compris la reproduction) est assujettie à sa politique d'utilisation que vous pouvez consulter en ligne.

https://apropos.erudit.org/fr/usagers/politique-dutilisation/ 


\title{
Les confréries de dévotion et l'éducation de la foi
}

\author{
Brigitte CAULIER \\ Université Laval, Histoire et CÉLAT
}

\begin{abstract}
Résumé
Les confréries de dévotion recrutant des adultes, telles que les congrégations mariales ou leurs copies, ont été des structures privilégiées pour soutenir l'éducation de la foi. Les exercices collectifs comportaient des périodes d'instruction et de prière ainsi que des séances d'apprentissage de la méditation. Le clergé qui dirigeait ces associations, recherchait une meilleure intériorisation de la foi et des principes de la religion chrétienne chez des laïcs déjà sélectionnés pour leur bonne conduite et leur piété.

Les directeurs profitaient des assemblées pour rafraîchir les connaissances doctrinales des associés et surtout pour leur rappeler leurs devoirs de chrétiens. Les sujets d'instructions, les lectures et les conseils donnés aux confrères et aux consæurs font apparaître la primauté accordée à la famille - parents comme enfants et même domestiques - dans l'épanouissement de la foi.
\end{abstract}

Les confréries de dévotion à encadrement strict, congrégations mariales jésuites ou inspirées de celles-ci, ont été implantées très tôt en NouvelleFrance, pour constituer «l'Amérique des dévots». Nous avons montré dans nos travaux antérieurs la pérennité de ces associations jusque dans les années 1960'. Nous examinerons ici concrètement quel support ces confréries ont fourni à l'éducation de la foi chez les adultes.

L'appartenance, librement consentie à une confrérie, avait pour but ultime - du moins en théorie - de faire progresser vers le salut. L'approfondissement de la foi et des connaissances religieuses a tenu un rôle essentiel dans certaines activités confraternelles. De plus, les congrégations

I Brigitte CAULIER, Les confréries de dévotion à Montréal, $17^{e}-19^{e}$ siècles, $\mathrm{PhD}$, Université de Montréal, 1986. Cette thèse a reçu l'appui du CRSH. 
mariales et leurs copies telles que la confrérie des dames de la SainteFamille ont encouragé leurs membres à privilégier la formation chrétienne familiale, prolongeant et renforçant ainsi l'enseignement catéchétique reçu à l'extérieur.

Les manuels de ces associations, les recueils d'instructions et les cahiers d'assemblées de leurs directeurs permettent d'envisager l'ampleur du travail éducatif accompli dans ce cadre. Trois exemples montréalais seront utilisés: une confrérie de recrutement mixte fondée en 1732, la Bonne-Mort, les Dames de la Sainte-Famille (1663) et la Congrégation des Hommes de Ville-Marie (1693).

\section{Parfaire l'éducation religieuse des consœurs et des confrères:}

L'entrée dans une confrérie signifiait, en partie, l'acceptation de persévérer dans la religion en participant à des exercices religieux plus nombreux que ceux imposés aux autres fidèles. Les pratiques individuelles et collectives ont contribué à soutenir la formation religieuse des adultes inscrits dans les confréries. Les directeurs ont développé cette formation non seulement par la prédication ou les lectures commentées, mais aussi par l'apprentissage de la prière et de la méditation, pendant les exercices collectifs, en incitant les associés à poursuivre ces pratiques à la maison, seuls ou en famille. Les assemblées, triduum et retraites, ainsi que les solennités étaient autant d'occasions de favoriser l'approfondissement de la foi.

\section{A. La prédication et la méditation dans les exercices collectifs:}

\section{Les assemblées}

Les confrères et consœurs se retrouvaient à intervalle régulier pour se recueillir ensemble. Ils se voyaient mensuellement à la Bonne-Mort. Les Dames de la Sainte-Famille s'assemblaient aux deux semaines le mardi à deux heures, prévenues par la cloche. Quant aux hommes, leur office était hebdomadaire. Comme pour la Bonne-Mort, les exercices collectifs de ces derniers se déroulaient sur toute l'anné, contrairement à la Sainte-Famille dont les vacances fluctuèrent.

Ces assemblées devaient, en principe, réunir tous les membres pour des exercices de dévotion bien précis, qui duraient en moyenne une heure pour la Bonne-Mort et la Sainte-Famille. La première comportait essentiellement la récitation du chapelet et une instruction du directeur:

On commencera l'assemblée, par le Veni Sancte Spiritus, l'on dira ensuite le chapelet de la Sainte-Vierge, après quoi on lira quelques uns de ces Statuts, ou quequ'autre Livre de piété, ou bien l'on fera une exhortation selon le tems (sic) et 
la commodité du Directeur de l'Association, après la lecture ou exhortation l'on dira le Chapelet des Morts et les Litanies de la bonne Mort, ensuite le Sub tuum praesidium ${ }^{2}$.

Les dames de la Sainte-Famille avaient des réunions très semblables qui débutaient par une invocation au Saint-Esprit, suivie du chapelet de la Sainte-Famille «avec la briève Méditation» dont le guide est reproduit dans le manuel. À cet exercice succédaient l'instruction du directeur ou l'explication du règlement d'une demi-heure, les avis divers et les recommandations de prières. L'assemblée se clôturait sur le De profondis en l'honneur des consœurs et des directeurs défunts.

La Congrégation se réunissait chaque dimanche, tôt le matin, pour réciter l'office de la Sainte-Vierge et entendre la messe. Celle-ci ne dispensait pas les membres de la messe dominicale à la paroisse. Après la récitation de l'office et «avant la messe le père spirituel fait une instruction ou donne les avis convenables, fait les annonces, et après la messe, il récite à haute voix les litanies de la Sainte-Vierge et les oraisons qui suivent»3. Un nocturne de l'office de la Sainte-Vierge s'intercalait entre celles-ci et le saint sacrifice. Les réunions de la Congrégation étaient donc les seules à inclure la messe, preuve d'une volonté de créer de bonnes habitudes chez les hommes pas toujours dociles. Cette pratique était néanmoins très particulière à Montréal et ne semble pas avoir existé ni au Québec ni en Europe. Elle justifiait, si elle était en usage dès la création, la méfiance des Sulpiciens, titulaires de la paroisse Notre-Dame.

Les réunions régulières devaient donc renforcer le sentiment d'appartenance à ces confréries et favoriser les progrès de la conversion individuelle de leurs associés.

\section{Triduum et retraites}

$\mathrm{Au} 19^{\mathrm{e}}$ siècle, pour mieux préparer les associés à la fête patronale de la Sainte-Famille et de la Congrégation, les directeurs organisent un triduum ou une retraite. Pour la Congrégation, nous ne possédons qu'une seule mention en 1855 qui en précise les dispositions financières. M. Roupe introduisit la pratique à la Sainte-Famille dix ans plus tôt. Ces exercices n'étaient pas obligatoires mais visaient à raffermir la piété et à faire de la solennité une véritable rénovation des âmes.

Le Triduum, c'est-à-dire un exercice de trois jours, pendant lesquels comme dans une espèce de retraite, elles se recueilleront et se disposeront, par la prière, la

2 Règlement de la confrérie de l'adoration perpétuclle du S. Sacrement et de la Bonne Mort, érigée dans l'église paroissiale de Ville-Marie en l'Isle de Montréal, en Canada, Montréal, F. Mesplet, 1776, p. 8. Désormais nous conserverons l'orthographe des auteurs.

3 Archives de la Société de Jésus au Canada français (ASJCF), 2274, Usages de M. Chicoisneau, p. 114. 
méditation et autres exercices à la réception des sacrements, et tacheront de se renouveller dans la ferveur et l'accomplissement fidèle de leurs saintes règles ${ }^{4}$.

Les dames devaient se rendre par deux fois à l'église de l'Hôtel-Dieu qui possédait les reliques de la Sainte-Famille, pour des exercices encadrant la journée de travail. «L'oraison en commun tous les matins à $6 \mathrm{~h} 1 / 2$ pendant une demie heure, qui sera suivi de la sainte messe à laquelle elles assisteront, et le soir à $51 / 2 \mathrm{~h}$ une instruction suivie du salut» ${ }^{5}$. Au total, les exercices du matin auxquels pouvait se joindre le public, comportaient aussi des avis sur des pratiques à mener dans la journée «pour celles des Dames qui auront plus de loisir», et la messe n'avait lieu qu'à 7 heures et demie. M. Roupe voyait dans cette retraite, car les termes se confondent pour lui, un moyen de faire pratiquer les exercices qu'il propose aux femmes: «Expliquer pourquoi je préfère donner une instruction en forme d'oraison, c'est que leur ayant donné il y a deux ans plusieurs instructions sur la manière de faire l'oraison, je veux leur en montrer la pratique» 6 .

\section{B. La lecture personnelle et commentée:}

Pour remédier aux désordres possibles, les directeurs de la SainteFamille et de la Congrégation ont cru en vertu de l'édification par le bon livre, tout comme ils redoutaient les effets des «mauvais livres».

\section{Encouragement à la lecture}

Depuis les origines de la confrérie, $\mathrm{M}^{\mathrm{gr}}$ de Laval avait misé sur la lecture pour le progrès spirituel des dames de la Sainte-Famille: «Elles tascheront d'avoir quelque livre de dévotion, dont elles liront ou feront lire tous les jours tant qu'il sera possible, en présence des enfans et domestiques»?.

La Congrégation, formée de lisants comme l'exigent les règlements, mena jusqu'au bout la démarche en fondant sa propre bibliothèque en 1835 . Celle-ci était accesssible aussi au public, premier pas vers une bibliothèque paroissiale qui verra le jour, en juillet 1844 , sous la forme de l'Oeuvre des bons livres. Cette dernière reçut de la Congrégation quelque six cents volumes. La gestion d'un tel organisme incluant le prêt public ayant entraîné des difficultés financières et des besoins en personnel trop lourds, la confrérie dut le céder à l'Oeuvre fondée par M. Quiblier. L'initiative demeure

\footnotetext{
4 Archives de la Compagnie de Saint-Sulpice de Montréal (ACSSM), carton 167 ,
} Cahiers d'assemblées de la Sainte-Famille (CASF), 1825-48, p. 210, 8 avril 1845.

5 Ibid., p. 210.

6 Ibid., p. 210.

7 La solide dévotion à la très sainte famille, de iesus, marie et ioseph avec un catéchisme qui enseigne à pratiquer leurs vertus, Paris, Florentin Lambert, 1675, p. 27. 
intéressante car elle révèle le rôle moteur dévolu par le clergé aux associations pieuses, dans la mise en place de l'encadrement laïc.

\section{La prédominance du livre de piété}

Le livre, particulièrement au $19^{\mathrm{e}}$ siècle, connaît un statut ambivalent; il représente aux yeux des clercs un potentiel subversif très fort, et d'aucuns préféreraient des ignorants à des pervertis par les mauvaises idées. Le directeur de la Sainte-Famille, en 1854, invitait les dames à se «défier des livres et des lectures», «Défiez-vous de tout ce qu'on peut dire et imprimer. Priez». À défaut de pouvoir interdire la lecture, les directeurs des confréries tentaient, au $19^{\mathrm{e}}$ siècle, de l'orienter vers des ouvrages conformes à la doctrine et à la morale.

Aussi ont-ils recommandé presqu'exclusivement des ouvrages à caractère religieux, au demeurant très nombreux à cette époque en Europe et qui alimentent les bibliothèques ecclésiastiques canadiennes-françaises. La Congrégation des Hommes se conforme à ce modèle lorsqu'elle fonde sa bibliothèque: «qu'il seroit établi immédiatement dans la sacristie de la chapelle de la dite congrégation une bibliothèque composée d'un choix de livres dogmatiques et moraux»8.

La gamme des ouvrages de ce genre s'élargit à cette époque, elle se compose aussi bien des "grands classiques» du 17e que de la production contemporaine. Dans la première catégorie, les préparations à la mort les plus célèbres sont fortement recommandées aux dames de la SainteFamille et aux congréganistes pendant tout le $19^{\mathrm{e}}$ siècle. En 1848, M. Roupe engageait les dames à réciter le chapelet pour les âmes du purgatoire, qui se trouvait dans L'Ange conducteur, ouvrage du père Coret, édité pour la première fois en 1662 et best-seller à l'époque. Le manuel de piété des congréganistes conseillait, pour approfondir la méditation, de recourir à L'âme élevée à Dieu de Barthélémi Beaugrand (également auteur de L'âme pénitente ou le nouveau pensez-y bien), ainsi que le Pensez-y bien de Paul de Barry, puis des ouvrages comportant aussi des exercices préparatoires, comme L'Évangile médité, les Considérations $d u$ mois dans la Journée du Chrétien, les Instructions de la Jeunesse de P. H. Humbert, le Combat spirituel, l'Introduction à la vie dévote, l'Imitation de Jésus Christ. Ouvrages dont se nourrissaient aussi les directeurs des confréries dans leur prédication et dans leur propre vie spirituelle. Ces ouvrages ont connu un regain de popularité en Europe au même moment.

8 Archives de la Fabrique de Notre-Dame de Montréal (AFNDN), Registre des délibérations du conseil et réceptions des congréganistes (RDCR), 1835-86, Grand Conseil 20 novembre 1835 . 
Ces directeurs bénéficiaient d'une production contemporaine nombreuse et de qualité très inégale venant principalement de France. On encourageait la lecture ainsi que l'abonnement à des périodiques catholiques tels que La Semaine religieuse ou même les Annales de Lourdes, Les cahiers d'assemblées tenus par les directeurs de la Sainte-Famille pendant tout le $19^{\mathrm{e}}$ siècle révèlent la diversité des lectures suggérées ou faites en assemblées. Histoires édifiantes et classiques ultramontains ont été diffusés par le canal confraternel. La présence de Sulpiciens français renforçait cette influence. ('est ainsi que M̀. Mãartineau a pubiicisé avec succès ceite littérature de piété française auprès des dames de la Sainte-Famille et des nombreuses associations qu'il a dirigées à Notre-Dame de Montréal. Ses talents d'orateur, bien connus dans la paroisse, n'ont pas été étrangers à sa réussite. La société de charité des dames de la Sainte-Famille a laissé dans ses archives le témoignage de ses succès: «Mr le Directeur nous fit beaucoup de plaisir, en reprenant la lecture et le commentaire de nos chères Paillettes d'or"'. Toutefois, il savait alterner les historiettes extraites de la Semaine religieuse de Toulouse avec les textes de $\mathrm{M}^{\mathrm{gr}} \mathrm{de}$ Ségur sur la messe, de ${ }^{\mathrm{gr}}$ Dupanloup, du Cardinal Pie et de Charles de Montalembert ainsi que de $\mathrm{M}^{\mathrm{gr}}$ Gaume. Il retenait surtout de ces auteurs ultramontains leurs ouvrages de piété, au-delà des divisions qui les opposèrent entre eux.

La lecture commentée des Saintes-Écritures demeurait le privilège des directeurs dans le cadre des assemblées et lors des solennités particulières aux confréries. Les grandes étapes de l'année liturgique donnaient lieu à des instructions axées sur les Écritures. Le recours à la Bible a beaucoup varié selon les directeurs et les associations. Certains suivaient de près le calendrier liturgique et s'appuyaient ainsi beaucoup plus sur les Écritures. Les six années d'instructions de M. Sauvage aux associés de la Bonne-Mort ont été très inspirées par la vie liturgique et restent très proches de la prédication dominicale. $M$. Sauvage ne s'éloigne pas de ses sermons destinés à la paroisse lorsqu'il s'adresse à la confrérie assemblée, à la fin des années 1830 (1836-1841). À la Sainte-Famille, M. Martineau décida de commenter les livres de la Genèse et de l'Exode, tout en l'agrémentant de considérations générales sur la création, tel un professeur de sciences naturelles. De novembre 1874 à juillet 1880, il instruisit, par cycles, les dames de la Sainte-Famille des grands épisodes de l'Histoire sainte, depuis le récit de la création jusqu'à la mort de Moïse, en se servant des Causeries sur l'Ancien et le Nouveau Testament, d'Eugène de Margerie, publiées en 1870. Cette référence aux Saintes-Écritures passe par le

9 ACSSM, carton 167. Comptes rendus des dépenses de la Société de charité de la Sainte-Famille, 1871-75, assemblée du 20 janvier 1875. 
biais d'un ouvrage de littérature populaire, recours fréquent pour animer les assemblées de cette confrérie.

Ce choix révèle, en partie, la spécificité des activités confraternelles par rapport aux pratiques religieuses, comme la messe dominicale. Elles devaient s'en démarquer pour éviter toute forme de concurrence ou d'ambiguïté pouvant inciter les laïcs à relâcher leur assiduité à la messe. De même, le directeur se devait de rendre les assemblées attrayantes en se distançant des règles de la prédication dominicale. Mais les buts demeurent identiques. Toutes les activités confraternelles collectives ou individuelles doivent favoriser la progression des associés dans la foi en raffermissant leurs connaissances religieuses et leur expérience spirituelle. Elles se veulent aussi le moyen de susciter les bons comportements.

\section{Les confréries comme écoles de formation doctrinale et morale}

Les associations confraternelles ont contribué à consolider l'acquis du catéchisme chez les adultes. Même si les directeurs insistent sur la mise en pratique des principes chrétiens appris au catéchisme et sur les démonstrations de piété, il n'en demeure pas moins que la mise à jour des connaissances doctrinales trouvait sa place lors des instructions et des lectures, à l'occasion, bien souvent, des fêtes liturgiques.

\section{A. Raffermir les connaissances doctrinales}

Celles-ci donnaient lieu à une préparation pendant les assemblées précédentes. Le temps du Carême et la fête de Pâques, par exemple, tout comme l'Avent et Noël, étaient marqués par des instructions s'y rapportant.

En 1872, la pénitence et l'explication de la messe nourrissaient les propos de M. Martineau aux dames de la Sainte-Famille, auxquelles il lisait et commentait l'ouvrage de $\mathrm{M}^{\mathrm{gr}}$ de Ségur sur la messe. De février à juin, il évoque ainsi les différents types de sacrifice: «Le sacrifice, sacrifices sanglants - leurs raisons. Combien de figuratifs principaux: Isaac - l'Agneau Pascal, La vache rousse, Les deux tourterelles - Les bons sacrifices non sanglants: Pain, vin, huile, sel, encens, jamais de miel»10. Suivent plusieurs reprises dans les instructions des directeurs de la Sainte-Famille, chaque moment de la messe étant l'objet d'explications minutieuses. ${ }^{4}$

10 ACSSM, carton 168, ACSF, 1866-85, 12 mars 1872. 
La présence réelle faisait aussi l'objet de commentaires à la SainteFamille et à la Bonne-Mort, dont le but premier consistait à soutenir la dévotion au Saint-Sacrement. Ces instructions, suivies sur plusieurs semaines, permettaient aux directeurs de faire le bilan sur des points de doctrine essentiels. De plus, ils ne se contentaient pas de rappeler les grandes solennités ou d'annoncer les exercices de dévotion s'y rattachant, ils en expliquaient la signification, comme en 1856 à la Sainte-Famille:

Jeudi saint, institution de la Sainte Eucharistie pour être avec nous toujours Sacerdoce quels biens. St Sacrifice de ia messe. Suivons ces 5 jours cians ses stations NS JC comme Marie comme Magdeleine. St Jean, St Nicodème. St Joseph d'Arimathie, Stes femmes et non comme les juifs aveugles endurcis ${ }^{11}$.

Les associés bénéficiaient ainsi d'une préparation accrue aux célébrations religieuses, ainsi que d'une meilleure compréhension des rituels que le reste des paroissiens. Mais il s'agissait ultimement pour les confrères et les consœurs d'imiter Jésus-Christ dans leur vie quotidienne.

\section{B. La conversion morale des membres}

Toute confrérie doit contribuer au salut de ses membres en aidant chacun d'eux et en permettant l'entraide mutuelle, comme l'évoque M. Sauvage aux confrères de la Bonne-Mort. L'association soutient le laïc dans sa lutte contre le démon:

\footnotetext{
Ainsi nous voyons un saint Basil et saint Grégoire, à leurs exemples il y a toujours eus dans le monde des personnes vertueuses qui se sont réunis ou fait des sociétés particulières pour sexiter a la vertu pour s'encourager a être fidels a la religion, à la pieté ${ }^{2}$.
}

Les critères moraux de sélection étant déjà élevés, les directeurs de ces confréries orientent les exigences morales et le travail sur soi en fonction de la vie quotidienne des associés.

\section{Le rappel constant des devoirs d'état}

S'adressant à des adultes, les directeurs des trois associations n'ont de cesse de leur répéter leurs devoirs d'état. Ceux-ci impliquent à ce niveau, des responsabilités sociales et familiales en accord avec le statut de chrétien.

Parmi les devoirs de tout congréganiste, énoncés dans le manuel de piété de 1882, figurent «l'attachement aux obligations de son état» ainsi que «l'amour de Dieu, du prochain et des ennemis, la justice envers tous»13. Ce qui se traduit sur le plan social et politique par «une parfaite

11 ACSSM, carton, 168, ACSF, 1848-64,p. 110, 18 mars 1856, Granjon.

12 ACSSM, dossier $31, \mathrm{n}^{\circ} 78,5$ août 1837.

13 Manuel de piété à l'usage des Congréganistes de la Sainte Vierge de Ville-Marie, Montréal, J.B. Rolland et Fils, 1882, p. 335. 
obéissance aux lois et aux autorités». L'encadrement des hommes par les confréries était à cet égard très important afin de les détourner, au $19^{\mathrm{e}}$ siècle, des mouvements syndicaux mais aussi du libéralisme politique et religieux, incarnés aux yeux du clergé dans la franc-maçonnerie, épouvantail menaçant à Montréal et motif majeur d'exclusion de la Congrégation: "Seront pareillement exclus tous ceux qui se seraient affiliés à quelque société secrète, défendue comme telle par l'autorité ecclésiastique» ${ }^{14}$. Les congréganistes étaient encouragés à respecter l'ordre établi. Les oraisons pour le salut du S. Sacrement comportaient une prière pour l'évêque et une autre pour le roi.

Remplir les devoirs de son état permet surtout de se sanctifier, comme M. Sauvage le développe dans une instruction aux associés de la Bonne-Mort:

Sommes nous maitres traitons nos inferieurs avec douceur avec justisse et bonté. Regardons les comme des freres comme des enfants de dieu. Faisons au prochain ce que nous desirerions qu'on nous fasse a nous même. Sommes nous serviteurs avons nous des maîtres respectons l'auctorité qu'ils ont sur nous comme venant de dieu soyons fidels a nos obligations envers eux non seulement en leur presence et lorsque nous sommes vus mais en leur apsence ${ }^{15}$.

Les critères d'admission à la Sainte-Famille portaient essentiellement sur la bonne conduite. Or «l'esprit de la Sainte-Famille consiste à imiter les sacrées personnes qui la composent, chacun selon son estat et condition» 16 , et les femmes à qui l'on reconnaissait la lourde tâche de l'éducation, se devaient d'entraîner mari et enfants à une vie dévote, et "sanctifier les mariages», état dangereux s'il en fut, et inférieur au célibat. Ce thème est donc récurrent dans les trois associations et traverse les siècles, il sous-tend un projet religieux d'encadrement laïc qui repose sur la structure familiale.

\section{Un climat familial paisible}

La prédication des directeurs s'adressant à des consœurs de la SainteFamille ou de la Bonne-Mort insiste sur le rôle fondamental de la mère dans le maintien d'un climat familial serein favorisant la sanctification du mariage. La soumission et l'humilité représentent aux yeux des directeurs les deux grandes qualités qui garantissent celle-ci.

Les dames de la Sainte-Famille, sur le modèle de la Vierge, devaient être de bonnes épouses, soumises au mari qui était le chef. $\mathrm{M}^{\mathrm{gr}}$ de Laval comptait sur elles pour la conversion de leur foyer:

\footnotetext{
14 lbid., p. xvii, article 16.

15 ACSSM, dossier 31, n 83, 3 février 1838.

16 La solide dévotion..., p. 22.
} 
Envers le mari, nous rappelle le règlement réédité sans modification au $19^{\mathrm{c}}$ siècle, un amour sincere et cordial, qui fasse qu'on aye un grand soin de tout ce qui le regarde, selon le temporel, et le spirituel taschant toujours de le gagner à Dieu par prieres, bons exemples, et autres moyens convenables; un grand respect, obéissance, douceur et patiente à souffrir ses défauts, et mauvaises humeurs ${ }^{17}$.

L'humilité apparaît comme la seule vertu capable d'apporter la paix dans les ménages, car elle éloigne emportements et irritabilité, la plaie des familles, que M. Sauvage tente bien souvent de corriger: «une femme qui dans son ménage née veüt rien suporter qui s'impatiente pour peu de chose qui ne cherche pas a faire aimer sa maison sa société a son mari ne parviendra jamais par les reprimandes à se faire ecouter» 18 . Les mères doivent se faire aimer de leurs enfants, afin d'être plus persuasives dans leurs remontrances. L'humilité «maintien la paix l'union dans les familles et avec les voisins qui souvent ne sont attiré que par l'esprit d'orgeuil qui veut toujours avoir raison et l'emporter sur les autres» ${ }^{19}$. Le directeur de la Bonne-Mort exhorte les femmes, qui composent l'essentiel de son auditoire, à éviter de donner prise aux médisances, mais aussi à savoir les recevoir: «elle nous porte a suporter avec patience les injures et à les pardonner et par la a les rendre meritoires devant dieu». À leur origine, l'orgueil, principal vice, selon M. Sauvage, auquel on doit opposer son contraire qui «met un obstacle à l'ambition naturel aux orgueilleux de s'elever au dessus du prochain». Tous les défauts viennent de cet «amour de soi-même», tout ce que l'humilité doit apaiser, c'est l'orgueil qui l'excite:

Or, clame M. Sauvage, quels sont les fautes que vous avez le plus souvent a vous reprocher des impatiences occasionnées soit par vos enfants, vos engagez etc des complaisance sur vous meme en comparant votre conduite avec celle des autres quelques medisances dans vos conversations. La source de toute ces miseres c'est l'orgueil 20 .

Les mères de famille ont donc la lourde responsabilité de conduire dans le droit chemin mari et enfants. Les prédicateurs de Notre-Dame les considéraient aussi très facilement responsables des mésententes conjugales et familiales, de la débauche du mari et du libertinage des enfants — thème prisé et ressassé de la solennité de la Sainte-Famille -

Aussi sainte Monique est-elle l'exemple rappelé constamment aux dames de la Sainte-Famille puisqu'elle réussit à convertir son mari païen, au surplus très violent, et son fils pécheur. Au $19^{\mathrm{e}}$ siècle, les directeurs de la confrérie rappelaient aux dames, les jours de la retraite des hommes

\footnotetext{
17 Ibid., p. 22-23.

18 ACSSM, dossier $31, n^{\circ} 80$, f. 1 , verso.

19 Ibid., recto.

20 ACSSM, dossier 31, n 105, Instruction 16, ler février 1841.
} 
avant Noël: «Je recommande à vos prières le succès de la retraite des hommes qui va commencer dans quelques jours. Combien chacune doit désirer la sanctification de son mari» 21 .

Harmoniser la vie familiale devait favoriser la conversion de chaque membre, y compris les domestiques. L'éducation de la foi passe, sans aucun doute pour les directeurs, par un environnement familial exemplaire. La confrérie représente un moyen de plus pour inciter les parents à assumer pleinement leurs responsabilités dans l'éducation chrétienne de leurs enfants. Les arguments de ce discours ne différaient guère de ceux utilisés en chaire, mais ils revenaient souvent et leur application pouvait en être mieux contrôlée.

\section{Former des parents responsables de l'éducation religieuse de leurs enfants}

\section{A. Les devoirs des parents en matière d'éducation religieuse}

\section{Les devoirs paternels}

Les congréganistes auxquels on demande de donner le bon exemple, doivent s'appliquer à faire régner la piété dans leur famille. Leurs devoirs concernent particulièrement leur descendance, pour laquelle la formation religieuse passée supplante la scolaire, et aucune mention ne concerne précisément leur femme:

Une attention particulière, quant aux pères de famille, à veiller sur leurs enfants, à leur donner une éducation religieuse et honnête, à faire régner la piété et la paix dans leurs familles, afin d'édifier leurs concitoyens, et d'attirer sur leurs maisons les bénédictions du ciel22.

Dans la prédication sulpicienne de la solennité de la Sainte-Famille, un seul sermon s'adresse particulièrement aux hommes, celui de $M$. Roques qui l'a utilisé en 1803 et 1819 . Il y rappelle les devoirs et obligations des pères vis-à-vis de leurs enfants et domestiques, tout en leur rappelant la hiérarchie de leur modèle: «vous tenez la place de dieu, vous devez donc encore plus pourvoir au besoin spirituel de ceux qui... vous appliquez a faire regner dans vos maisons Dieu dont vous etes les lieutenants» 23 . Même si une nuance modère le propos «ne pensez pas que dieu vous ait donné l'autorité uniquement pour vos interets», le modèle choisi comme figure du père est Dieu, et non saint Joseph, suivant le parallèle habituel avec la Sainte-Famille. Les exigences imposées aux femmes étaient plus lourdes.

\footnotetext{
21 ACSSM, carton 168, CASF, 1848-64, p. 140, 15 décembre 1857, Rousselot.

22 Manuel de piété..., 1882, p. xii.

23 ACSSM, dossier 29, n 29, A. Roques, 1803, 1819.
} 


\section{Les devoirs maternels}

Après avoir veillé à la sanctification de leur mari, les femmes doivent favoriser l'éclosion de la foi et son maintien chez leurs enfants. Les modèles qui leur sont donnés à méditer comme sainte Monique, ou sainte Jeanne de Chantal ont servi d'exemple aussi bien à leur époux qu'à leurs enfants. Depuis la fondation de la confrérie, l'état de mère s'associe indissolublement à celui de chrétienne, car c'est à la femme qu'est confiée l'éducation religieuse des enfants:

À l'égard des enfans, un grand soin de les élever dans la crainte de Dieu, de leur apprendre et faire dire tous les jours les prières; leur inspirer une grande horreur du péché; ne leur souffrir rien ou Dieu soit offensé sans les chastier; une grande douceur à les corriger! la patience à souffrir leurs petites foiblesses, envisageant sans cesse dans leurs personnes celle du petit lesus, dont ils sont les images vivantes» 24 .

Le règlement de la confrérie édité en 1675 exprime une vision nuancée de l'éducation où l'enfant commence à être reconnu dans sa spécificité et non comme petit adulte. Le catéchisme qui prolonge et explicite le règlement, insiste sur la manière de «corriger» les enfants: «on est obligé de le faire avec zele soubs peine de se rendre coupable de leurs fautes, mais jamais par pure colere, car quand la passion accompagne la correction, bien loin d'estre utile aux enfans ils en deviennent plus mechans»25. Remarque de bon sens, mais néanmoins assez neuve et propre aux milieux aristocratiques européens de l'époque, qui avait la chance de pénétrer plus avant la société par le biais de la confrérie.

Toutefois, les cahiers des directeurs, deux siècles plus tard, insistent beaucoup plus sur «l'horreur du péché» à enseigner aux enfants et sur leur éloignement «des mauvaises compagnies, surtout des serviteurs», que recommandait déjà le catéchisme de la Sainte-Famille. Le danger absolu pour les enfants, s'il faut en croire M. Martineau, qui est le plus explicite, ce sont les vacances, car l'oisiveté suscite le vice et la saison estivale mérite condamnation à ses yeux: «un mot sur la saison: molesse, lacheté, fraicheur». Les spectacles qui les agrémentent, dont le théâtre, ne trouvent aucune grâce à ses yeux et pour lui «La corruption va croissant exemples cités devant moi ces jours derniers. La vigilance maternelle». Dans les dernières années de son mandat, le Sulpicien fulminait contre ces dames et leur reprochait de ne pas favoriser l'éducation: «à qui la confiez-vous? Vous allez au rabais en tout». À la rentrée de 1884, son instruction porte sur «vos enfants et l'école la santé — vie de règle; l'instruction — vie d'étude; la vertu - vie de piété, favorisez ces trois choses»26. Il est le seul

24 La solide dévotion..., 1675, p. 23.

25 Ibid., catéchisme, p. 156.

26 ACSSM, carton 168, CASF, 1866-85, 16 septembre 1884. 
à avoir insisté autant sur ce point; auparavant dans les années 1850 , les directeurs recommandaient aux prières des dames, les premières communions et le catéchisme: «le Bien qu'il fait, malheur d'ignorer sa religion suggestion aux Dames de prier et d'y envoyer tous ceux qu'elles pourront» ${ }^{27}$. Vers cette même époque, les directeurs sollicitent les mères afin qu'elles entraînent leurs filles vers les Enfants de Marie ou la Congrégation des demoiselles, ainsi que la Sainte-Enfance. Et nous voyons que très rapidement ces regroupements ont adopté la retraite annuelle pour soutenir l'élan. L'Avent était ainsi consacré à la réflexion et à la conversion pour toutes les classes d'âge: «Recommander $1^{\circ}$ la Retraite qui se fait à l'Hôpital général pour les enfants de Bon Secours et autres demoiselles de la ville. $2^{\circ}$ la Sainte-Enfance afin que les parents fassent autant d'associés qu'ils ont d'enfants» 28 .

Les retraites des jeunes gens étaient aussi annoncées, et c'est par le relais de la Sainte-Famille que M. Martineau tenta de fonder un nouveau mouvement pour les enfants en janvier 1886: «Je me propose une Euvre si vous voulez l'adopter. Les petits enfants soulagés par les petits enfants. Apprendre à vos enfants l'économie, la mortification et la charité»29. Il semblerait que l'idée ait trouvé un répondant car une année plus tard, il mentionne que

Nos petits orphelins ont commencé: nos Demoiselles du St Nom de Marie sont venues aujourd'hui avec 40 piastres. Nous irons à notre tour porter notre tribut à $\mathrm{Mr}$ le Supérieur. Beauté de cette manifestation, en face de l'acharnement des ennemis de la religion 30 .

Les associations les plus souvent mentionnées concernent les filles qui apparaissent comme les plus menacées mais aussi les plus perméables aux bons principes; or si elles sont toutes sages, la débauche sera évitée aux garçons. Cette préoccupation, si elle se développe avec M. Martineau, a fait l'objet de remarques bien avant lui, mais de façon sporadique. Ainsi M. Sattin notait pour l'assemblée du 21 juin 1831: «Avis général à donner pour la décence dans les maisons au sujet des habillements soit pour les Dames, soit pour leurs demoiselles»31. M. Roupe, par la suite, ne manqua pas de renouveler cette recommandation qui correspondait encore parfaitement à l'esprit du règlement et du catéchisme de la Sainte-Famille qui indique nettement que le vêtement doit favoriser la décence mais surtout rester le reflet exact de la condition sociale de l'enfant qui le porte: «elles doivent prendre garde de ne pas ajuster leurs enfants d'une manière

\footnotetext{
${ }^{27}$ ACSSM, carton 168, CASF, 1848-64, p. 99, 2 octobre 1855, Granjon.

28 Ibid., p. 87, 5 décembre 1854, Nercam.

29 ACSSM, carton 168, CASF, 1885-87, 21 janvier 1886.

30 Ibid., 11 janvier 1887.

31 ACSSM, carton 167, CASF, 1845-48, p. 91.
} 
très affectée, vaine et au dessus de leur condition, ce qui nourrit en eux un esprit de vanité qu'il n'est pas aisé de leur oster quand ils sont plus grands»32. Les interdits se précisent en fait dans les années 1850 avec la condamnation épiscopale de la Sainte-Catherine:

De jeudi en 8,25 de ce mois, jour de ste Catherine, vous vous rappelerer les avis que nous vous avons donnés, dimanche dernier, de la part de Monseigneur, pour vous détourner vous et vos enfans des divertissemens dangereux, par lesquels on prophane ce jour-là ${ }^{33}$.

En 1884 , Mí. Martineau, qui n’avaii pás mànưué de rapppeler cctitc fête, en profite pour raconter en parallèle la vie de Marguerite Bourgeoys et celle de sainte Catherine et encourager ainsi «l'instruction et l'éducation chrétienne des jeunes filles». Thème qui lui était cher et qu'il avait développé au cours d'un cycle d'instructions consacrées aux devoirs des dames, dès son arrivée en octobre 1866, et qu'il avait agrémentées des écrits de Fénelon.

\section{B. Une piété familiale propice à l'éveil religieux des enfants}

C'est dans l'intimité familiale que les enfants s'initient doucement aux pratiques religieuses dès avant leur catéchisation. Ils pouvaient ainsi se familiariser avec les prières dans le cadre de la récitation du chapelet recommandée aux dames de la Sainte-Famille: «Elles réciteront tous les jours le chapelet en commun, dans leurs maisons, ou en leur particulier ne le pouvant en commun, en l'honneur de Iésus, Marie, Ioseph»34.

Les plus zèlées pouvaient y ajouter celui de la Sainte-Famille composé de trois dizaines en souvenir des trente ans de vie commune du saint modèle. L'importance accordée au Rosaire correspondait aux nouvelles orientations prises depuis le Concile de Trente qui valorisait le culte marial, cherchant à l'imposer même aux hommes. Or il est bien recommandé de le réciter en commun; les femmes doivent encourager la ferveur de leur maisonnée. La confrérie a suscité un culte domestique voulant favoriser à la fois, la piété mais aussi l'harmonie familiale.

Ainsi, $\mathrm{M}^{\mathrm{gr}}$ de Laval avait prévu que les dames se recueillent devant une gravure de la Sainte-Famille:

Elles auront dans leurs maisons quelque image de la Sainte-Famille devant laquelle elles feront leurs prières soir et matin à genoux, et renouvelleront tous les jours la donation et consécration qu'elles luy ont fait d'elles-mesmes, de leur mary, de leur enfans, et de toutes leur Famille, et porteront tant qu'elles pourront leur mary à faire de mesmes ${ }^{35}$.

\footnotetext{
32 La solide dévotion..., 1675, p. 154.

33 ACSSM, carton 168, CASF, 1848-64, p. 67, 16 novembre 1852.

${ }^{34}$ La solide dévotion..., 1675, p. 26.

35 Ibid., p. 26.
} 
La gravure devait être certainement l'une des deux qui ont été fixées au registre principal et qui représentent, pour l'une, la vie quotidienne du divin modèle, le père et son fils s'affairant à une charpente et la mère à l'aiguille, pour l'autre, plus abstraite, les trois personnes auréolées en oraison autour d'une table sous le faisceau de la colombe.

Les directeurs encourageaient la création d'un autel domestique audessus duquel l'image sainte servait de retable; celle-ci changeait selon les fêtes. En 1858, M. Rousselot inscrit dans son cahier: «mois de Marie inviter les Dames à suivre ses exercices - et à orner chez elle dans leur oratoire la statue ou l'image de la Sainte-Vierge.» $M^{\text {gr }}$ de Laval y avait vu l'endroit par excellence de l'intercession:

Elles y auront recours [à l'image], en toute leurs necessitez, afflictions, tentations, et aux occasions ou elles auront besoin de l'assistance du Ciel, demandant avec confiance, estant asseurées d'obtenir ce qu'elles demanderont par l'intercession de la Sainte Famille.

Lorsque M. Sattin reprit en main la confrérie devenue exsangue, il s'empressa de rétablir la coutume:

Je me suis arrangé, dit-il, avec les sœurs de l'Hôpital général pour en faire tirer une centaine de copies qui seront enluminées et ensuite encadrées sous des vitres, afin qu'elles servent d'ornements pour les appartements où elles seront placées ${ }^{36}$.

En 1826 et 1827 , ils revint plusieurs fois sur le thème et ensuite M. Roupe prendra le relais entre 1835 et 1848 , au moins une fois l'an.

Ces recommandations ne semblent pas avoir été données à la Congrégation, les livres de comptes restent muets sur de telles dépenses; c'était aux femmes de servir de catalyseur spirituel dans leur foyer pour une pratique plus sentie de la religion; aussi leur recommande-t-on de faire leurs exercices en commun ainsi que la lecture.

L'orientation familiale de la piété ne se limite pas à la seule confrérie de la Sainte-Famille. Le clergé mise, particulièrement au $19^{\mathrm{e}}$ siècle, sur la cellule familiale et l'on connaît les thèses ultramontaines qui confirment ou justifient ce choix. Les associations de laïcs fondées par les Jésuites se prêtaient facilement à cette orientation, puisqu'elles avaient tendu très tôt à la réalisation d'un idéal de famille chrétienne, analysée en Europe par Louis Châtellier ${ }^{37}$ jusque dans ses prolongements au $19^{\mathrm{e}}$ siècle.

Ainsi, les confréries de dévotion, congrégations mariales ou inspirées par celles-ci, ont constitué un moyen, privilégié par le clergé, d'encadrement des adultes. Elles ont servi de complément à leur formation

36 ACSSM, carton 168, CASF, 1825-48, 23 août 1825, p. 10.

37 Louis Châtellier, L'Europe des dévots, Paris, Flammarion, 1987. 
religieuse et représentent, en quelque sorte, une forme de catéchisme de persévérance, pour un petit nombre d'individus sélectionnés devant servir d'exemple à la paroisse. L'intervention des confréries se situe plus, toutefois, au niveau des comportements et des agirs que de l'apprentissage de connaissances comme le fait le catéchisme. Les liens avec la catéchèse demeurent à approfondir sur une plus large échelle; il semble déjà évident qu'une partie de l'effort de sensibilisation des parents à la nécessité de l'éducation religieuse de leurs enfants a été menée par le canal des confréries, en mettant i'accent sur i importance du ciimai famiiiai dans l'éclosion de la foi chez les enfants. 